\title{
Vertically coupled double quantum rings at zero magnetic field
}

\author{
F. Malet, M. Barranco, E. Lipparini,* R. Mayol, and M. Pi \\ Departament ECM, Facultat de Física and IN ${ }^{2}$ UB, Universitat de Barcelona. Diagonal 647, 08028 Barcelona, Spain \\ J. I. Climente \\ CNR-INFM S3 Center, Via Campi 213/A, 41100 Modena, Italy \\ J. Planelles \\ Departament CC.EE., Universitat Jaume I. 12080 Castelló, Spain
}

(Received 15 February 2006; revised manuscript received 20 April 2006; published 20 June 2006)

\begin{abstract}
Within local-spin-density functional theory, we have investigated the "dissociation" of few-electron circular vertical semiconductor double quantum ring artificial molecules at zero magnetic field as a function of interring distance. In a first step, the molecules are constituted by two identical quantum rings. When the rings are quantum mechanically strongly coupled, the electronic states are substantially delocalized, and the addition energy spectra of the artificial molecule resemble those of a single quantum ring in the few-electron limit. When the rings are quantum mechanically weakly coupled, the electronic states in the molecule are substantially localized in one ring or the other, although the rings can be electrostatically coupled. The effect of a slight mismatch introduced in the molecules from nominally identical quantum wells, or from changes in the inner radius of the constituent rings, induces localization by offsetting the energy levels in the quantum rings. This plays a crucial role in the appearance of the addition spectra as a function of coupling strength particularly in the weak coupling limit.
\end{abstract}

DOI: 10.1103/PhysRevB.73.245324 PACS number(s): 73.21.-b, 85.35.Be, 73.22.-f, 71.15.Mb

\section{INTRODUCTION}

Semiconductor quantum dots (QDs) are widely regarded as artificial atoms with properties similar to those of "natural" atoms. One of the most appealing properties is the capability of forming molecules. Systems composed of two QDs, QD artificial molecules (QDMs), coupled vertically, have been investigated experimentally and theoretically at zero magnetic field $(B)$, or submitted to magnetic fields applied in different directions, see e.g., Refs. 1-17 and references therein.

Semiconductor ring structures have also received considerable attention in connection with the Aharonov-Bohm effect, ${ }^{18}$ and the energy spectrum of nanoscopic selfassembled quantum rings occupied by few electrons has been experimentally analyzed. ${ }^{19}$ Recently, high-quality quantum rings (QRs) have been fabricated on a $\mathrm{AlGaAs}-\mathrm{GaAs}$ heterostructure containing a two-dimensional (2D) electron gas, by nanolithography with a scanning force microscope, see Refs. 20 and references therein. These studies have allowed researchers to extend previous studies to manyelectron nanoscopic rings, and have provided an experimental determination of the spin ground states of the rings by Coulomb-blockade spectroscopy, as well as the clear identification of a singlet-triplet transition, and the size of the exchange interaction matrix element; ${ }^{21}$ properties that had been also determined in the past for QDs. ${ }^{22}$

Very recently, two different types of nanometer-sized QR complexes have been realized. One such complex consists of two concentric QRs grown by droplet epitaxy on an $\mathrm{Al}_{0.3} \mathrm{Ga}_{0.7} \mathrm{As}$ substrate. ${ }^{23}$ The other complex consists of stacked layers of InGaAs/GaAs QRs, whose optical and structural properties have been characterized by photolumi- nescence spectroscopy and by atomic force microscopy, respectively. ${ }^{24,25}$ Motivated by these recent experimental works, we have undertaken a theoretical study, within localspin-density functional theory (LSDFT), of the ground state (gs) properties of QR's complexes at $B=0$. In this work, we present the results we have obtained for the case of two vertically coupled GaAs QRs, leaving aside for a separate study the case of concentric double QRs, whose phenomenology is somewhat different. ${ }^{26}$ To some extent, our work parallels the one we have carried out in the past for double QDs, with the aim of describing the electronic properties of QR molecules (QRMs) and discussing the similarities and differences between vertical QDM and QRM structures.

This work is organized as follows. In Sec. II, we present the method used to describe QRs and vertically coupled QRMs. The results we have obtained are discussed in Sec. III and a brief summary is presented in Sec. IV.

\section{LSDFT DESCRIPTION OF QUANTUM RINGS AND VERTICAL QUANTUM RING MOLECULES}

We closely follow the method of Ref. 27, where the interested reader may find it described in some detail. We recall that within LSDFT, the gs of the system is obtained by solving the Kohn-Sham (KS) equations. The problem is simplified by the imposed axial symmetry around the $z$ axis, which allows one to write the single particle (sp) wave functions as $\phi_{n l \sigma}(r, z, \theta, \sigma)=u_{n l \sigma}(r, z) e^{-l l \theta} \chi_{\sigma}$ with $n=0,1,2, \ldots, l$ $=0, \pm 1, \pm 2, \ldots$, with $-l$ as the projection of the sp orbital angular momentum on the symmetry axis.

We have used effective atomic units $\hbar=e^{2} / \epsilon=m=1$, where $\epsilon$ is the dielectric constant, and $m$ is the electron effective mass. In units of the bare electron mass, $m_{e}$, one has 
$m=m^{*} m_{e}$. In this system, the length unit is the effective Bohr radius $a_{0}^{*}=a_{0} \epsilon / \mathrm{m}^{*}$, and the energy unit is the effective Hartree $H^{*}=H^{*} / \epsilon^{2}$. In the numerical applications, we have considered GaAs, for which we have taken $\epsilon=12.4$, and $m^{*}$ $=0.067$. This yields $a_{0}^{*} \sim 97.9 \AA$ and $H^{*} \sim 11.9 \mathrm{meV}$.

In cylindrical coordinates, the KS equations read

$$
\begin{aligned}
& {\left[-\frac{1}{2}\left(\frac{\partial^{2}}{\partial r^{2}}+\frac{1}{r} \frac{\partial}{\partial r}-\frac{l^{2}}{r^{2}}+\frac{\partial^{2}}{\partial z^{2}}\right)+V_{c f}(r, z)+V^{H}+V^{x c}+W^{x c} \eta_{\sigma}\right]} \\
& \quad \times u_{n l \sigma}(r, z)=\varepsilon_{n l \sigma} u_{n l \sigma}(r, z),
\end{aligned}
$$

where $\eta_{\sigma}=+1(-1)$ for $\sigma=\uparrow(\downarrow), V_{c f}(r, z)$ is the confining potential, $V^{H}(r, z)$ is the direct Coulomb potential, and $V^{x c}$ $=\partial \mathcal{E}_{x c}(n, m) /\left.\partial n\right|_{g s}$ and $W^{x c}=\partial \mathcal{E}_{x c}(n, m) /\left.\partial m\right|_{g s}$ are the variations of the exchange-correlation energy density $\mathcal{E}_{x c}(n, m)$ in terms of the electron density $n(r, z)$ and of the local spin magnetization $m(r, z) \equiv n^{\uparrow}(r, z)-n^{\downarrow}(r, z)$ taken at the gs.

As usual, $\mathcal{E}_{x c}(n, m) \equiv \mathcal{E}_{x}(n, m)+\mathcal{E}_{c}(n, m)$ has been built from three-dimensional (3D) homogeneous electron gas calculations. This yields a well-known ${ }^{28}$ simple analytical expression for the exchange contribution $\mathcal{E}_{x}(n, m)$. For the correlation contribution $\mathcal{E}_{c}(n, m)$, we have used the parametrization proposed by Perdew and Zunger. ${ }^{29}$

For a double QR the confining potential, $V_{c f}(r, z)$ has been taken parabolic in the $x y$ plane with a repulsive core around the origin, plus a symmetric double quantum well of width $w$ each, in the $z$ direction. The potential in the $x y$ plane has circular symmetry, and in terms of the cylindrical coordinate $r$ it is written as

$$
V_{c f}(r)=V_{0} \Theta\left(R_{0}-r\right)+\frac{1}{2} m \omega_{0}^{2}\left(r-R_{0}\right)^{2} \Theta\left(r-R_{0}\right),
$$

with $\Theta(x)=1$ if $x>0$ and zero otherwise. The convenience of using a hard-wall confining potential to describe the effect of the inner core in QR's is endorsed by several works in the literature. ${ }^{31}$ We have taken $R_{0}=5 \mathrm{~nm}, w=5 \mathrm{~nm}, V_{0}$ $=350 \mathrm{meV}$, and $\omega_{0}=15 \mathrm{meV}$. The depth of the double quantum well is also $V_{0}$. This set of parameters fairly represents the smallest rings synthetized in Ref. 32, and together with the distance $d$ between constituent quantum wells, determine the confining potential. The distance $d$ is varied to describe QRMs at different inter-ring distances. For the single "thick" QRs, we will discuss, as a reference system that we have used the same confining potential in the $x y$ plane, together with a single quantum well in the $z$ direction. For all structures, the sharp potential wells have been slightly rounded off, as shown in Ref. 30. Details about how the KS and Poisson equations have been solved can be found in Ref. 27 .

\section{RESULTS AND DISCUSSION}

\section{A. Single QR}

We have carried out calculations for a single thick QR confined, as indicated in the previous section, and for a strictly 2D QR confined by the radial potential Eq. (2), as indicated, e.g., in Ref. 33. These results will help us to discuss the appearance of the addition spectra of the QRM.

Figure 1 shows the addition energies $\Delta_{2}(N)$

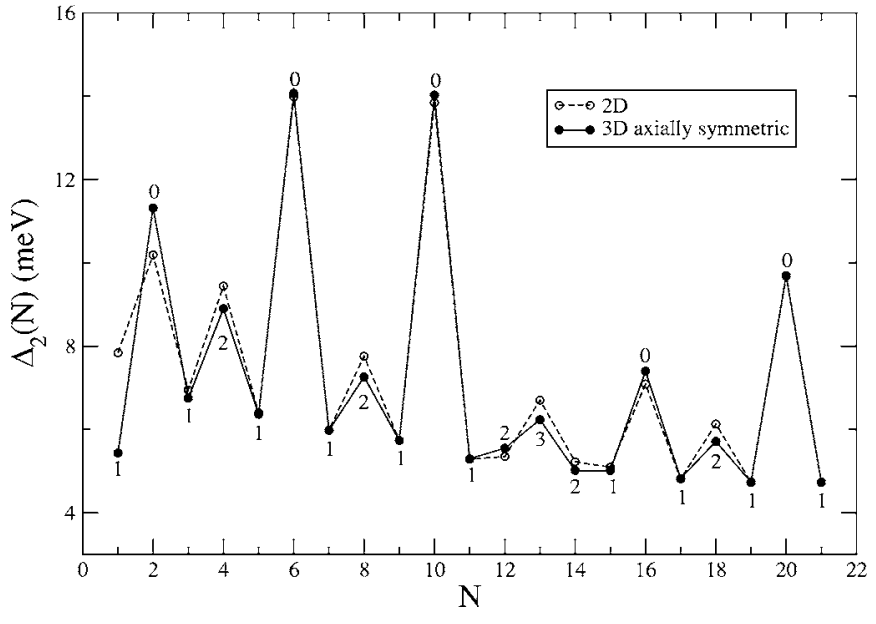

FIG. 1. Addition energies $\Delta_{2}(N)(\mathrm{meV})$ as a function of the number of electrons $N$ for a thick QR (solid dots, solid lines) and a strictly 2D QR (open dots, dashed lines). The value of $2 S_{z}$ obtained for the thick QR is indicated.

$$
\Delta_{2}(N)=E(N+1)-2 E(N)+E(N-1),
$$

where $E(N)$ is the total energy of the $N$ electron $\mathrm{QR}$, as a function of $N$. It can be seen that the 2D and "thick"-i.e., axially symmetric 3D-models sensibly yield the same results for this observable, a well-known result for QDs. ${ }^{13}$ For the thick rings, the value of the calculated total spin third component, $2 S_{z}$, is also indicated in the figure. We want to point out that in the $N=3$ case, the $2 \mathrm{D}$ model configuration is fully polarized $\left(2 S_{z}=3\right)$. This is due to the fact that the exchange-correlation energy is overestimated by strictly $2 \mathrm{D}$ models. ${ }^{34}$ Fully polarized $N=3$ QR configurations are not an artifact of the LSDFT. As a matter of fact, they have been also found by exact diagonalization methods for some ring sizes and confining potential choices. ${ }^{35}$ For all of the configurations displayed in Fig. 1 but $N=3$, both models yield the same spin assignments.

The gs spin assignments we have found here coincide with those of Ref. 33, although the height of the peaks in $\Delta_{2}(\mathrm{~N})$ depends to a large extent on the confining potential. They are related to the relative stability of the electronic shell closures in the ring, which for $N>6$ are substantially different from these of QDs. In the case of rings, they are mainly governed by the four-fold degeneracy of the noninteracting sp levels with $|l| \neq 0$, and the two-fold degeneracy of the noninteracting sp levels with $|l|=0$. This yields the marked shell closures at $N=2,6,10,20$, and 28 with $S_{z}=0$, as well as the $S_{z}=0 \mathrm{gs}$ found for $N=24$. The $2 S_{z}=2 \mathrm{gs}$, that regularly appear between them, indicate that Hund's rule is fulfilled by single QRs.

The complex spin structure around $N=13$ deserves some comments. It is due to the occupancy of the second $(s \uparrow)$ state with $l=0$-this spin structure is missing in other QR calculations that employ a different confining potential. ${ }^{36}$ Figure 2 displays the sp energies $\varepsilon_{n l \sigma}$ for $N=13$, which are distributed paraboliclike as a function of $l$, each parabola corresponding to a different value of the principal quantum number $n$. This 


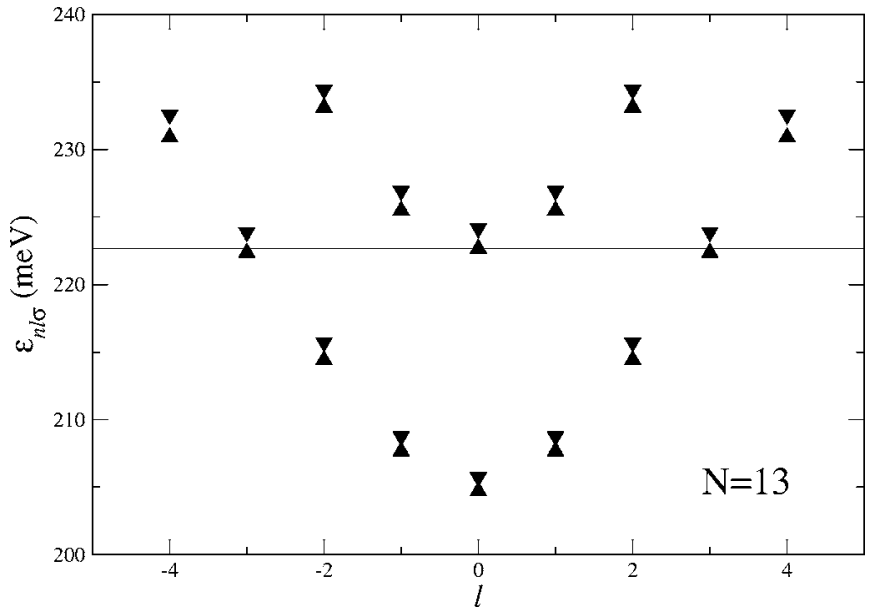

FIG. 2. Single particle energy levels (meV) as a function of $l$ for a thick QR with $N=13$. Upward (downward) triangles denote $\uparrow(\downarrow)$ spin states. The thin horizontal line represents the Fermi energy.

figure explains the filling sequence around $N=13$. For $N$ $=12$, the second $(0 \uparrow)$ state is empty, yielding $2 S_{z}=2$; for $N$ $=13$ the exchange interaction favors the filling of this state yielding $2 S_{z}=3$; for $N=14$, one of the $( \pm 3 \downarrow)$ states is filledthey are degenerate-yielding $2 S_{z}=2$ [actually, this manyelectron configuration is nearly degenerate with the one in which the $(0 \downarrow)$ state is filled instead, which also yields $\left.2 S_{z}=2\right]$. For $N=16$, the $(0 \downarrow)$ and $( \pm 3 \downarrow)$ become populated, producing a fairly strong shell closure.

\section{B. Homonuclear QRMs}

We consider first the case of a QRM formed by two identical QRs. By analogy with natural molecules, we call them homonuclear QRM. We have calculated their gs structure for $d=2,4$, and $6 \mathrm{~nm}$, and up to $N=32$. For a given electron number $N$, the evolution of the gs ("phase") of a QRM as a function of $d$ may be thought of as a dissociation process. ${ }^{13}$ Within LSDFT, each sp molecular orbital has, as quantum labels, the third component of the spin and of the orbital angular momentum, the parity, and the value of reflection symmetry about the $z=0$ plane. Symmetric states $|S\rangle$ are called bonding states, and antisymmetric states $|\mathrm{AS}\rangle$ are called antibonding states.

The energy splitting between bonding and antibonding sets of sp states, $\Delta_{\text {SAS }}$, can be properly estimated ${ }^{13}$ from the energy difference of the antisymmetric and symmetric states of a single electron QRM, $\Delta_{\mathrm{SAS}} \sim E\left({ }^{2} \Sigma_{u}^{-}\right)-E\left({ }^{2} \Sigma_{g}^{+}\right)$—see below for the notation-and varies from $24.9 \mathrm{meV}$ at $d$ $=2 \mathrm{~nm}$ (strong coupling), to $1.49 \mathrm{meV}$ at $d=6 \mathrm{~nm}$ (weak coupling). In this range of inter-ring distances, $\Delta_{\mathrm{SAS}}$ can be fitted as $\Delta_{\mathrm{SAS}}=\Delta_{0} e^{-d / d_{0}}$, with $\Delta_{0}=82 \mathrm{meV}$ and $d_{0}=1.68 \mathrm{~nm}$. The relative value of the two energies $\hbar \omega_{0}$ and $\Delta_{\text {SAS }}$ crucially determines the structure of the molecular phases along the dissociation path.

Figure 3 shows the evolution with $d$ of the gs energy and molecular phase of a QRM made of $N=3-7$ electrons. $^{37}$ Each configuration is labeled using an adapted version of the ordinary spectroscopy notation; ${ }^{6}$ namely, ${ }^{2 S+1} L_{g, u}^{ \pm}$, where $S$ is

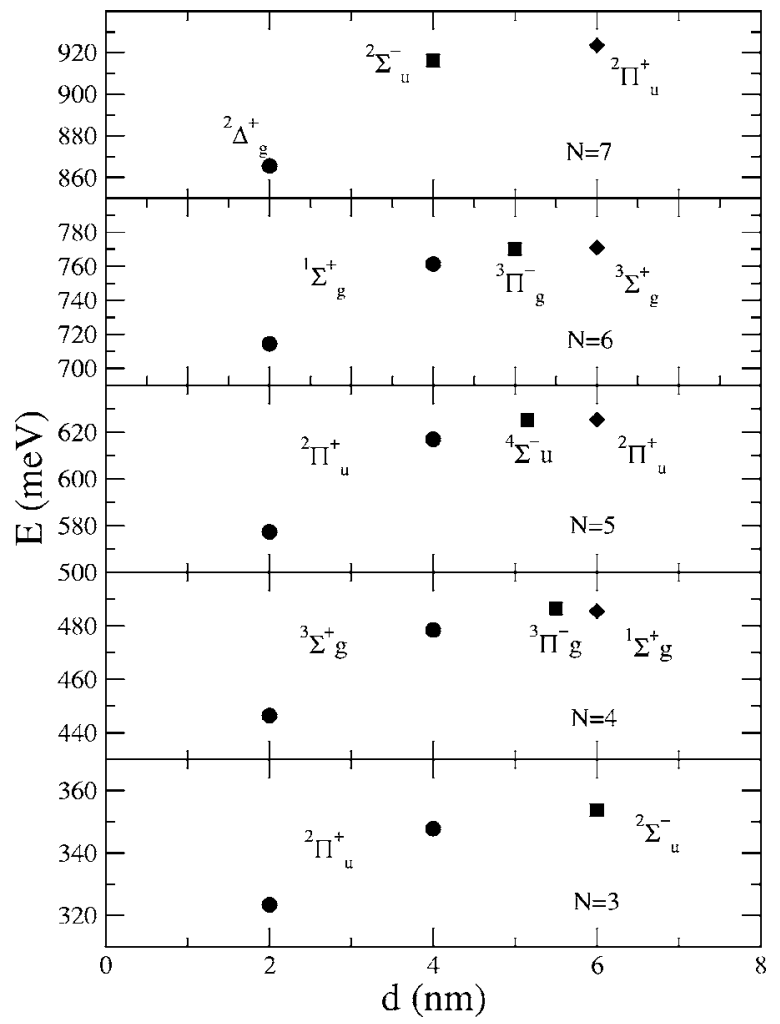

FIG. 3. Energy (meV) and gs molecular phases of the homonuclear QRM as functions of the inter-ring distance $d$ for $N=3-7$. For a given QRM, different phases are represented by different symbols. For example, in the $N=3$ case, dots correspond to ${ }^{2} \Pi_{u}^{+}$ gs's and the square to a ${ }^{2} \Sigma_{u}^{-}$gs, whereas for $N=4$, dots correspond to ${ }^{3} \Sigma_{g}^{+}$gs's, the square to a ${ }^{3} \Pi_{g}^{-}$gs, and the diamond to a ${ }^{1} \Sigma_{g}^{+}$gs.

the total $\left|S_{z}\right|$, and $L$ is the total $\left|L_{z}\right|$. The superscript $+(-)$ refers to even (odd) states under reflection with respect to the $z=0$ plane, and the subscript $g(u)$ refers to positive (negative) parity states. To label the molecular sp states, we have used the standard convention of molecular physics, using $\sigma, \pi, \delta, \ldots$ if $l=0, \pm 1, \pm 2, \ldots$. Uppercase Greek letters are used for the total $\left|L_{z}\right|$. Figure 3 shows that the energy of the molecular phase increases with $d$. This is due to the increase of the energy of the sp bonding states as $d$ increases, ${ }^{27}$ that dominates over the decrease in Coulomb energy. At larger inter-ring distances (not shown in the figure), the constituent QRs are so apart that eventually the decrease of Coulomb energy dominates and the tendency is reversed. The phase sequences are the same as for double QDs, ${ }^{13}$ although the transition inter-ring distances, which obviously depend on the kind and strength of the confining potential, are different. As for double QDs, we have found that the first phase transition of a few-electron QRM is always due to the replacement of an occupied bonding sp state by an empty antibonding one.

Figure 4 shows the addition spectra for homonuclear QRM up to $N=31$ for the three selected inter-ring distances. Also shown is the reference spectrum of a single QR. For small $d\left(\Delta_{\mathrm{SAS}} \gg \hbar \omega_{0}\right)$, the spectrum of the QRM is rather similar to a single $\mathrm{QR}$, especially for few-electron systems, with minor changes arising in the $N \sim 12$ and $\sim 24$ regions 


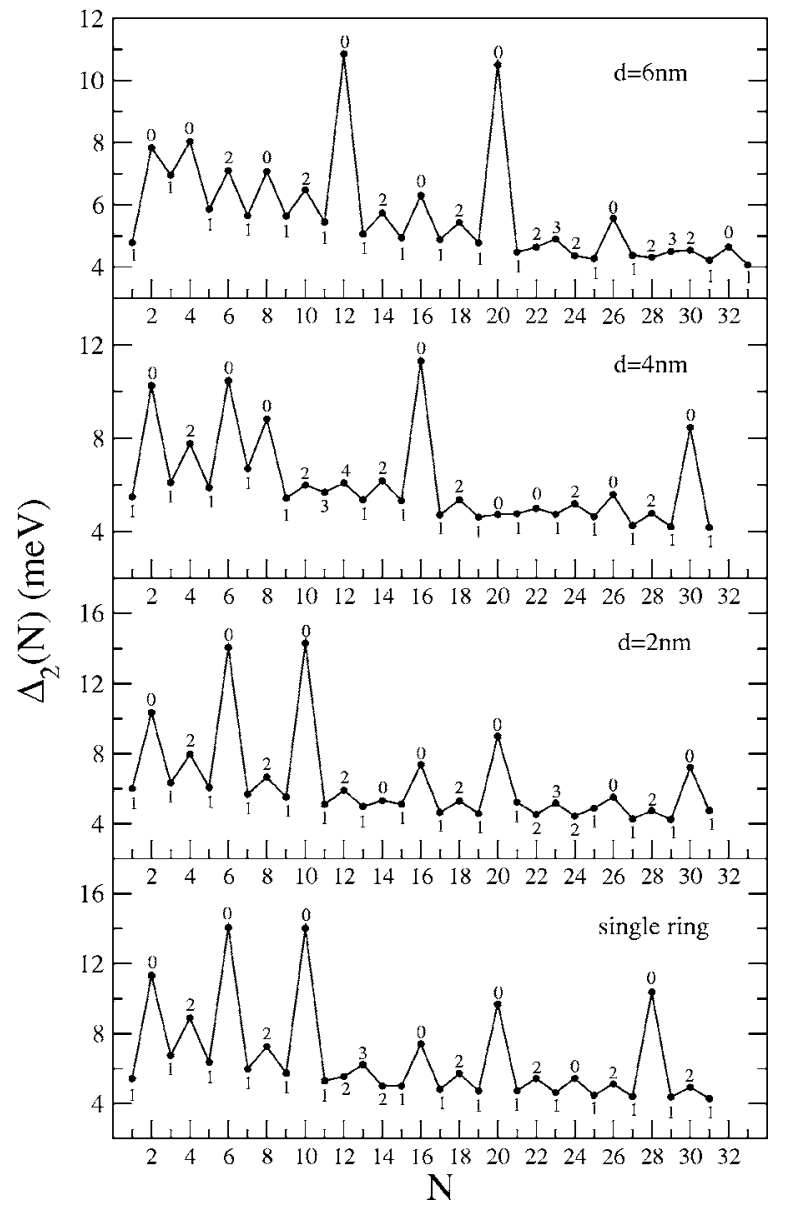

FIG. 4. $\Delta_{2}(N)$ for homonuclear QRM with inter-ring distances $d=2,4$, and $6 \mathrm{~nm}$. The addition energies have been offset for clarity. Also shown is the reference spectrum for a single $\mathrm{QR}$. The value of $2 S_{z}$ is indicated.

that will be commented upon below. It is clear that for $d$ $=2 \mathrm{~nm}$, the two QRs are electrostatically and quantummechanically coupled, and behave as a single system. At intermediate distances, the spectrum pattern becomes more complex; but at larger distances (e.g., $d=6 \mathrm{~nm}$ ), when the QRM molecule is about to dissociate, the physical picture that emerges is rather simple and can be interpreted using intuitive yet approximate arguments. At large distances $\left(\Delta_{\mathrm{SAS}} \ll \hbar \omega_{0}\right)$, the QRs are coupled only electrostatically, and most $|S\rangle$ and $|\mathrm{AS}\rangle$ states are quasidegenerate. Electron localization $^{38}$ in each constituent $\mathrm{QR}$ can be achieved by combining these states as $\left(|S\rangle_{ \pm}|\mathrm{AS}\rangle\right) / \sqrt{2}$; and as a consequence, the strong $S_{z}=0$ peaks found at $N=12$ and 20 are readily interpreted from the peaks appearing in the single $\mathrm{QR}$ spectrum at $N=6$ and 10 . The process can be viewed as the symmetric dissociation of the original QRM leading to very robust closed-shell single QR configurations. This is also the origin of the QRM $S_{z}=0$ peaks at $N=2$ and 4 . In the former case, the QRM configuration corresponds to one single electron being hosted in each constituent $\mathrm{QR}$ coupled into a singlet state; and in the latter case, the QRM configuration is viewed as two QRs, each one occupied by two electrons filling the $1 s$ shell.

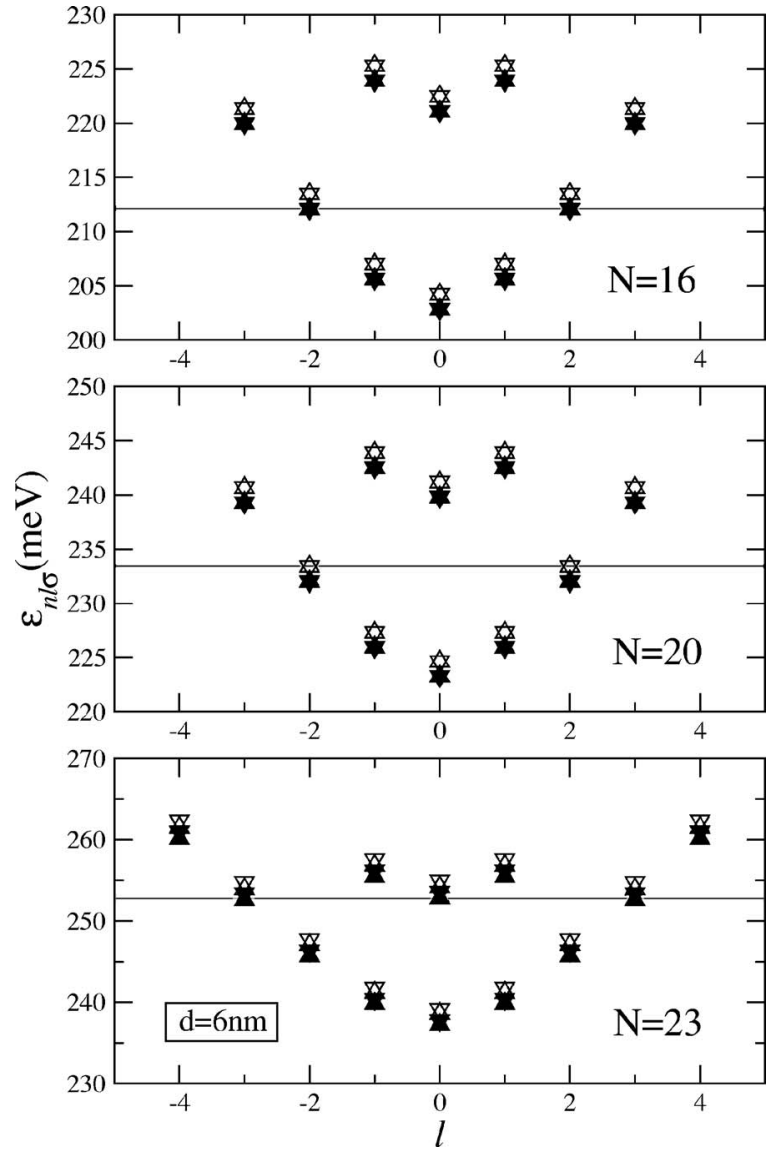

FIG. 5. Single particle energy levels $(\mathrm{meV})$ as a function of $l$ for an homonuclear QRM with $d=6 \mathrm{~nm}$ and $N=16$ (top panel), $N=20$ (middle panel), and $N=23$ (bottom panel). Upward (downward) triangles denote $\uparrow(\downarrow)$ spin states. Open (solid) triangles correspond to antibonding (bonding) states. The thin horizontal line represents the Fermi energy.

At $d=6 \mathrm{~nm}$, other dissociations display a more complicated pattern, such as $16 \rightarrow 8+8$, or $8 \rightarrow 4+4$, whose final products are QRs that fulfill Hund's rule, whereas the actual QRM has $S_{z}=0$. These could be interpreted as rather entangled QRM, "harder" to dissociate, for which a $d=6 \mathrm{~nm}$ inter-ring distance is not large enough to allow for electron localization. The quasidegeneracy of occupied $|S\rangle$ and $|\mathrm{AS}\rangle$ states at given $d$ plays a role in this intuitive analysis; whether or not the number of states is equal, they may eventually be combined to favor localization. An example of these two different situations is illustrated in Fig. 5, where we show the sp states of the $N=16,20$, and $23 \mathrm{QRM}$ at $d$ $=6 \mathrm{~nm}$. In the case of $N=16$ and 23, the filled bonding states near the Fermi level have not filled an antibonding partner and are delocalized in the whole volume of the QRM, contributing to the molecular bonding at that distance, whereas all other bonding states can be localized combining them with their antibonding partner. As in natural molecules, some orbitals contribute to the molecular bonding, whereas others do not.

\section{Heteronuclear QR molecules}

For vertically coupled lithographic double QDs, it has been found unavoidable that a slight mismatch is uninten- 
tionally introduced in the course of their fabrication from materials with nominally identical constituents quantum wells, ${ }^{13}$ which is responsible for electron localization as the interdot coupling becomes weaker. This offsets the energy levels in the QDs by a certain amount that was estimated to be up to $2 \mathrm{meV}$, and this plays a crucial role in the appearance of the addition energy spectra as a function of the coupling strength, particularly in the weak coupling limit. A similar picture is also found in coupled self-assembled QDs, where strain propagation between adjacent layers of dots often leads to top QDs of increased size. ${ }^{39}$

It is likely that the same fabrication limitations will appear in the case of vertically coupled double quantum rings. Anticipating this situation, we have carried out a series of QRM calculations in which the double quantum wells have the same width $w$ but slightly different depths; namely $V_{0} \pm \delta$, with $\delta \ll V_{0}$. It can be easily checked that in the weak coupling limit $\left(2 \delta \gg \Delta_{\mathrm{SAS}}\right), 2 \delta$ is approximately the energy splitting between the bonding and antibonding sp states, which would be almost degenerate if $\delta=0$. For this reason, we call the mismatch (offset) the quantity $2 \delta$.

We have considered two possible values of the mismatch, namely $2 \delta=2$ and $4 \mathrm{meV}$, and have obtained the corresponding addition spectra for up to $N=13$ electrons-according to our previous experience with double QDs ${ }^{13}$ we expect that the larger differences will arise in few-electron QRM. The results are displayed in Fig. 6. It can be seen that in the strong coupling limit, the effect of the mismatch on the addition energies is negligible, as expected. ${ }^{13}$ The electrons are completely delocalized in the whole volume of the QRM, and the introduced mismatch is unable to localize them in either of the constituents QRs.

The situation changes in the weak coupling limit. Indeed, for the few-electron QRM, which is the more interesting physical situation, we have shown before that the fingerprint of homonuclear character is the appearance, in this limit, of the peaks in the addition spectrum corresponding to $N=2$ and 4 , as well as their spin assignment $S_{z}=0$. It can be seen from Fig. 6 that in the intermediate regime $(d=4 \mathrm{~nm})$, the $N=4$ peak still corresponds to a $2 S_{z}=2$ configuration; but at larger inter-ring distances, it eventually disappears, yielding an addition spectrum that clearly manifests the heteronuclear character of the QRM and constitutes a clean fingerprint of these kind of configurations.

It is useful to display the dissociation of the QRM representing the $d$ evolution of the sp molecular wave functions, introducing the $z$-probability distribution function: ${ }^{13}$

$$
\mathcal{P}(z)=2 \pi \int d \operatorname{rr}[u(r, z)]^{2} .
$$

Examples of these probability functions can be seen in Fig. 7 , where we show $\mathcal{P}(z)$ for $N=20,2 \delta=4 \mathrm{meV}$, and $N=8$, $2 \delta=2 \mathrm{meV}$ (deeper well always in the $z<0$ region), each for the chosen three $d$ values. In each panel, the probability
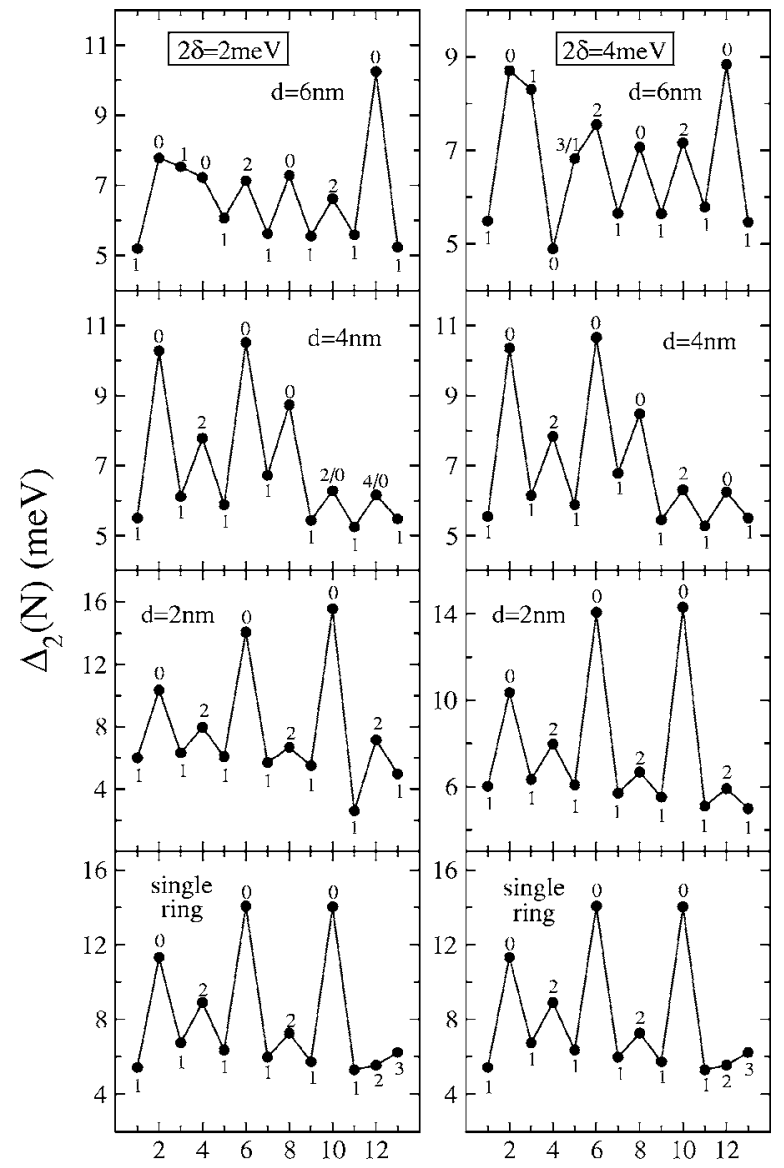

$\mathrm{N}$

FIG. 6. $\Delta_{2}(N)$ for heteronuclear QRM with inter-ring distances $d=2,4$, and $6 \mathrm{~nm}$ and mismatch $2 \delta=2 \mathrm{meV}$ (left panels) and $2 \delta$ $=4 \mathrm{meV}$ (right panels). The addition energies have been offset for clarity. Also shown is the reference spectrum for a single QR. The value of $2 S_{z}$ is indicated. Note that in some cases two different values of $S_{z}$ have been assigned to the same peak. This means that the corresponding configurations are nearly degenerated.

functions are plotted ordered from bottom to top according to the increasing sp energies. For $N=20$, the final configurations are the closed shell $N=10,2 S_{z}=0 \mathrm{QRs}$; whereas for $N=8$ the $N=4,2 S_{z}=2$ Hund's rule QR configurations emerge.

Finally, we discuss the case of two QRs of different radii vertically coupled to build an axially symmetric QRM, and study the effect that this asymmetry has on the addition spectrum (we have discarded a possible disalignment of the $\mathrm{QR}$ symmetry axes, as addressing this situation would require a much more demanding full $3 \mathrm{D}$ calculation) ${ }^{40}$ To this end, we have taken for one ring $R_{0}=6 \mathrm{~nm}$, while for the other one we have kept the same value as before, $R_{0}=5 \mathrm{~nm}$ ( $\delta$ is set to zero this case). Vertically coupled QD's of different radii have been described in Ref. 10 to address the sensitivity of the exchange coupling to the value of an in-plane applied electric field.

We show in Fig. 8 the addition spectra for up to $N=14$ electrons and $d=2,4$, and $6 \mathrm{~nm}$. It can be seen that in the strong and intermediate coupling cases they are fairly similar 

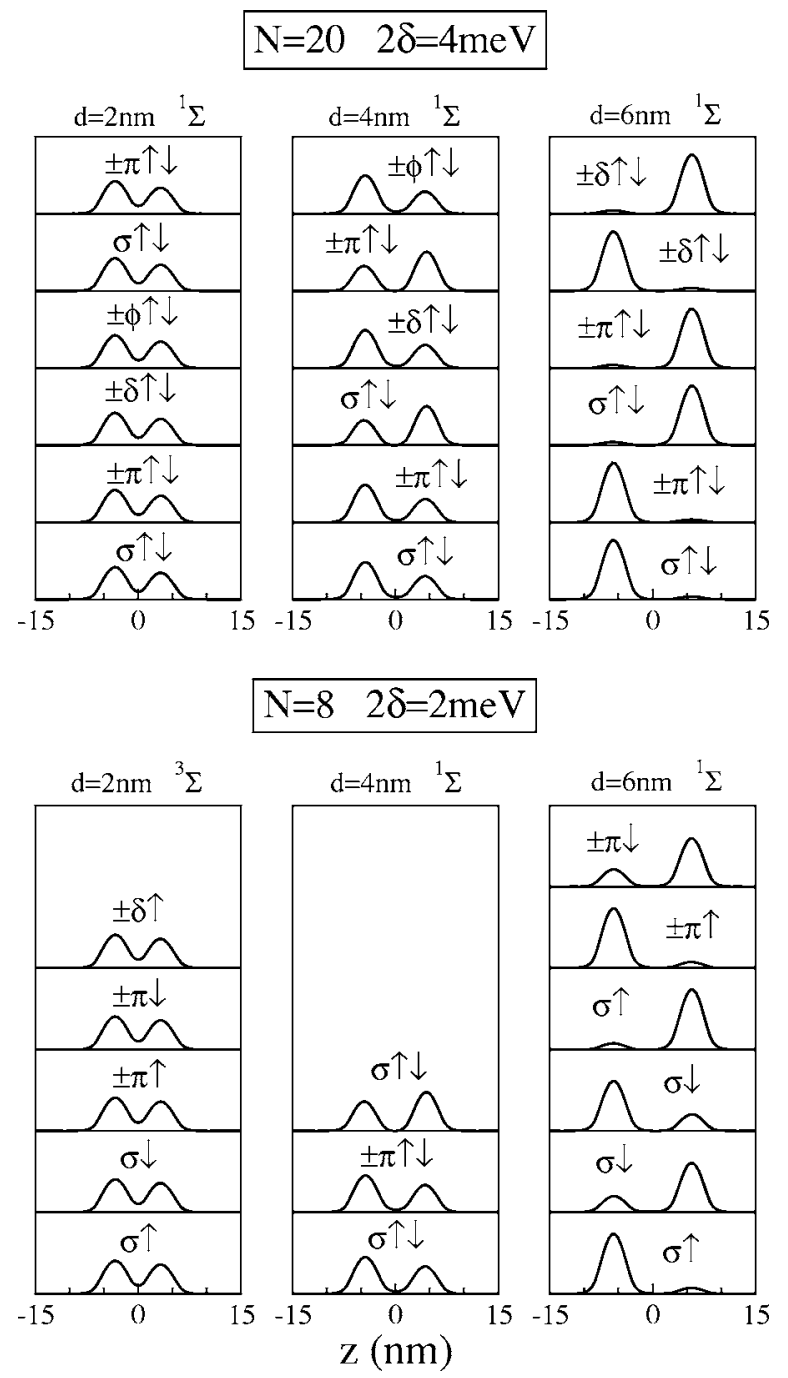

FIG. 7. Calculated probability distributions $\mathcal{P}(z)$ (arbitrary units) as a function of $z$ for heteronuclear QRMs with $N=20,2 \delta$ $=4 \mathrm{meV}$ (top panels), and $N=8,2 \delta=2 \mathrm{meV}$ (bottom panels). The corresponding molecular configuration is also indicated.

to the previous heteronuclear case- and to the homonuclear case as well-indicating a fairly robust structure of the QRM in these limits. As before, the heteronuclear character clearly shows up in the weak coupling limit, with a peak structure and $S_{z}$ assignments remarkably similar to those discussed in the previous situation with $\delta \neq 0$.

\section{SUMMARY AND OUTLOOK}

We have discussed the appearance of the addition energy spectra of homonuclear and heteronuclear QRMs at zero magnetic field. In particular, we have addressed the addition energy spectrum of QRM from the weak to the strong coupling limits. Fingerprints of homo- and heteronuclear molecular character have been pointed out in the weak coupling limit. As occurred in the study of vertically coupled double QDs, ${ }^{13}$ we believe this may be helpful in the analysis of future experiments on vertical QRMs. While some of the

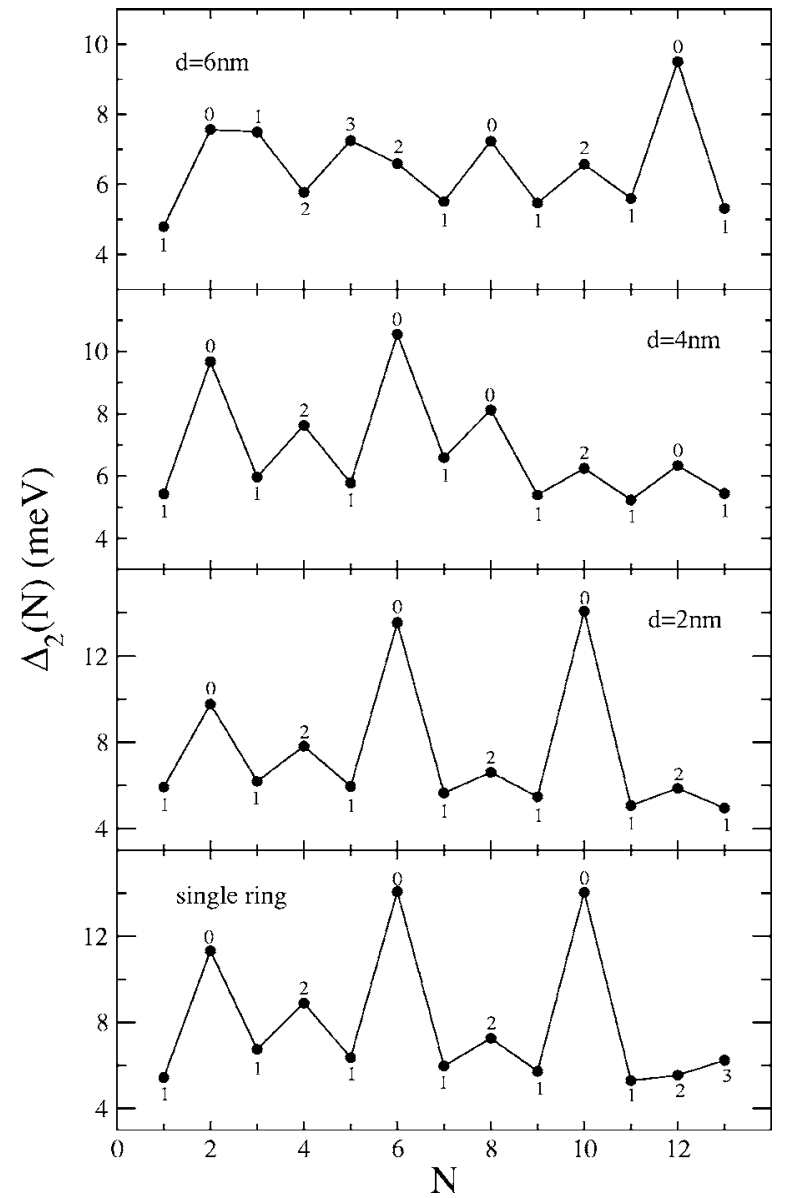

FIG. 8. $\Delta_{2}(N)$ for heteronuclear QRM made of QR with different core radii $R_{0}=5$ and $R_{0}=6 \mathrm{~nm}$, hosting up to $N=14$ electrons and inter-ring distances $d=2,4$, and $6 \mathrm{~nm}$. The addition energies have been offset for clarity. Also shown is the reference spectrum for a single QR. The value of $2 S_{z}$ is indicated.

effects we have described are common to QD and QR vertical molecules, e.g., the dominance of the single QD or QR physics in the strong coupling limit, the preference in the dissociation process of the channels that yield the more stable single QD or QR products (magic or Hund's rule fulfilling configurations), or the influence of mismatch in the addition spectrum in the weak coupling limit, our quantitative study allows us to associate realistic values to the physical magnitudes that characterize these effects in the case of QRMs, such as shell closures as a function of the inter-ring distance, as disclosed by the calculated addition spectra, and the gs spin assignments, for which we still lack of experimental results for comparison. This can be helpful in the analysis of future experiments on vertical QRMs.

The present study can be naturally extended to the case of QRMs submitted to magnetic fields of arbitrary direction. A rich interplay between molecular phases with different isospins is expected to appear as a function of $B,{ }^{15,30}$ which might have an observable influence on the Aharonov-Bohm effect and on the far-infrared spectroscopy of nanoscopic QRMs. 


\section{ACKNOWLEDGMENTS}

This work has been performed under Grants Nos. FIS2005-01414 from DGI (Spain), 2005SGR00343 from Generalitat de Catalunya, and CTQ2004-02315/BQU, UJIBancaixa Contract No. P1-B2002-01 (Spain). E. L. has been suported by DGU (Spain), Grant No. SAB2004-0091, and by CESCA-CEPBA, Barcelona, in the initial stages of this work through the program HPC-Europa Transnational Access. J.I.C. has been supported by the EU under the TMR network "Exciting."
*Permanent address: Dipartimento di Fisica, Università di Trento, and INFN, 38050 Povo, Trento, Italy.

${ }^{1}$ J. J. Palacios and P. Hawrylak, Phys. Rev. B 51, 1769 (1995).

${ }^{2}$ J. Hu, E. Dagotto, and A. H. MacDonald, Phys. Rev. B 54, 8616 (1996).

${ }^{3}$ G. S. Solomon, J. A. Trezza, A. F. Marshall, and J. S. Harris, Jr., Phys. Rev. Lett. 76, 952 (1996).

${ }^{4}$ O. Mayrock, S. A. Mikhailov, T. Darnhofer, and U. Rössler, Phys. Rev. B 56, 15760 (1997).

${ }^{5}$ Y. Asano, Phys. Rev. B 58, 1414 (1998).

${ }^{6}$ M. Rontani, F. Rossi, F. Manghi, and E. Molinari, Solid State Commun. 112, 151 (1999); M. Rontani, F. Troiani, U. Hohenester, and E. Molinari, ibid. 119, 309 (2001); M. Rontani, S. Amaha, K. Muraki, F. Manghi, E. Molinari, S. Tarucha, and D. G. Austing, Phys. Rev. B 69, 085327 (2004).

${ }^{7}$ P. Boucaud, J. B. Williams, K. S. Gill, M. S. Sherwin, W. V. Schoenfeld, and P. M. Petroff, Appl. Phys. Lett. 77, 4356 (2000).

${ }^{8}$ B. Partoens and F. M. Peeters, Phys. Rev. Lett. 84, 4433 (2000); Europhys. Lett. 56, 86 (2001).

${ }^{9}$ L. Martín-Moreno, L. Brey, and C. Tejedor, Phys. Rev. B 62, R10633 (2000); D. Sánchez, L. Brey, and G. Platero, ibid. 64, 235304 (2001).

${ }^{10}$ O. Mayrock, S. A. Mikhailov, T. Darnhofer, and U. Rossler, Phys. Rev. B 56, 15760 (1997).

${ }^{11}$ B. Jouault, G. Santoro, and A. Tagliacozzo, Phys. Rev. B 61, 10242 (2000).

${ }^{12}$ S. Amaha, D. G. Austing, Y. Tokura, K. Muraki, K. Ono, and S. Tarucha, Solid State Commun. 119, 183 (2001).

${ }^{13}$ M. Pi, A. Emperador, M. Barranco, F. Garcias, K. Muraki, S. Tarucha, and D. G. Austing, Phys. Rev. Lett. 87, 066801 (2001).

${ }^{14}$ P. Matagne, J. P. Leburton, D. G. Austing, and S. Tarucha, Phys. Rev. B 65, 085325 (2002).

${ }^{15}$ D. G. Austing, S. Tarucha, H. Tamura, K. Muraki, F. Ancilotto, M. Barranco, A. Emperador, R. Mayol, and M. Pi, Phys. Rev. B 70, 045324 (2004).

${ }^{16}$ D. Jacob, B. Wunsch, and D. Pfannkuche, Phys. Rev. B 70, 081314(R) (2004).

${ }^{17}$ M. Pi, D. G. Austing, R. Mayol, K. Muraki, S. Sasaki, H. Tamura, and S. Tarucha, in Trends in Quantum Dots Research, edited by P. A. Ling (Nova Science, 2005), p. 1.

${ }^{18}$ A. E. Hansen, A. Kristensen, S. Pedersen, C. B. Sorensen, and P. E. Lindelof, Phys. Rev. B 64, 045327 (2001).

${ }^{19}$ A. Lorke, R. J. Luyken, A. O. Govorov, J. P. Kotthaus, J. M. Garcia, and P. M. Petroff, Phys. Rev. Lett. 84, 2223 (2000).

${ }^{20}$ A. Fuhrer, S. Lüscher, T. Ihn, T. Heinzel, K. Ensslin, W. Wegscheider, and M. Bichler, Nature (London) 413, 822 (2001); T. Ihn, A. Fuhrer, T. Heinzel, K. Ensslin, W. Wegscheider, and M. Bichler, Physica E (Amsterdam) 16, 83 (2003).

${ }^{21}$ T. Ihn, A. Fuhrer, K. Ensslin, W. Wegscheider, and M. Bichler,
Physica E (Amsterdam) 26, 225 (2005).

${ }^{22}$ S. Tarucha, D. G. Austing, T. Honda, R. J. van der Hage, and L. P. Kouwenhoven, Phys. Rev. Lett. 77, 3613 (1996); S. Tarucha, D. G. Austing, Y. Tokura, W. G. van der Wiel, and L. P. Kouwenhoven, ibid. 84, 2485 (2000).

${ }^{23}$ T. Kuroda, T. Mano, T. Ochiai, S. Sanguinetti, K. Sakoda, G. Kido, and N. Koguchi, Phys. Rev. B 72, 205301 (2005).

${ }^{24}$ D. Granados, J. M. García, T. Ben, and S. I. Molina, Appl. Phys. Lett. 86, 071918 (2005).

${ }^{25}$ F. Suárez, D. Granados, M. L. Dotor, and J. M. García, Nanotechnology 15, S126 (2004).

${ }^{26}$ J. I. Climente, J. Planelles, M. Barranco, F. Malet, and M. Pi, cond-mat/0603588.

${ }^{27}$ M. Pi, A. Emperador, M. Barranco, and F. Garcias, Phys. Rev. B 63, 115316 (2001).

${ }^{28}$ S. Lundqvist, in Theory of the Inhomogenous Electron Gas, edited by S. Lundqvist and N. H. March (Plenum, New York, 1983), p. 149.

${ }^{29}$ J. P. Perdew and A. Zunger, Phys. Rev. B 23, 5048 (1981).

${ }^{30}$ F. Ancilotto, D. G. Austing, M. Barranco, R. Mayol, K. Muraki, M. Pi, S. Sasaki, and S. Tarucha, Phys. Rev. B 67, 205311 (2003).

${ }^{31}$ S. S. Li, and J. B. Xia, J. Appl. Phys. 89, 3434 (2001); A. Puente and Ll. Serra, Phys. Rev. B 63, 125334 (2001); J. I. Climente, J. Planelles, and F. Rajadell, J. Phys.: Condens. Matter 17, 1573 (2005).

${ }^{32}$ B. C. Lee and C. P. Lee, Nanotechnology 15, 848 (2004).

${ }^{33}$ A. Emperador, M. Pi, M. Barranco, and E. Lipparini, Phys. Rev. B 64, 155304 (2001).

${ }^{34}$ M. Rontani, F. Rossi, F. Manghi, and E. Molinari, Phys. Rev. B 59, 10165 (1999).

${ }^{35}$ J.-L. Zhu, S. Hu, Z. Dai, and X. Hu, Phys. Rev. B 72, 075411 (2005).

${ }^{36}$ J. C. Lin and G. Y. Guo, Phys. Rev. B 65, 035304 (2001).

${ }^{37}$ In order to catch all phases, we have carried out extra calculations at distances others than $d=2,4$, and $6 \mathrm{~nm}$ for some $N$ values.

${ }^{38} \mathrm{~A}$ thorough discussion of the localization properties of the molecular states for laterally coupled double quantum dots can be found in the review article by W. G. van der Wiel, S. De Franceschi, J. M. Elzerman, T. Fujisawa, S. Tarucha, and L. P. Kouwenhoven, Rev. Mod. Phys. 75, 1 (2003).

${ }^{39}$ N. N. Ledentsov, V. A. Shchukin, M. Grundmann, N. Kirstaedter, J. Böhrer, O. Schmidt, D. Bimberg, V. M. Ustinov, A. Yu. Egorov, A. E. Zhukov, P. S. Kop'ev, S. V. Zaitsev, N. Yu. Gordeev, Zh. I. Alferov, A. I. Borovkov, A. O. Kosogov, S. S. Ruvimov, P. Werner, U. Gösele, and J. Heydenreich, Phys. Rev. B 54, 8743 (1996).

${ }^{40}$ M. Pi, F. Ancilotto, E. Lipparini, and R. Mayol, Physica E (Amsterdam) 24, 297 (2004). 